\title{
Double strain state in a single GaN/AIN nanowire: Probing the core-shell effect by ultraviolet resonant Raman scattering
}

\author{
V. Laneuville, F. Demangeot, ${ }^{*}$ R. Péchou, P. Salles, and A. Ponchet \\ CNRS, CEMES (Centre d'Elaboration de Matériaux et d'Etudes Structurales), 29 Rue J. Marvig, BP 94347, F-31055 Toulouse, France \\ Université de Toulouse, UPS, 29 Rue J. Marvig, F-31055 Toulouse, France
}

G. Jacopin, L. Rigutti, A. de Luna Bugallo, M. Tchernycheva, and F. H. Julien

Institut d'Electronique Fondamentale, Université Paris Sud XI, UMR 8622 CNRS, F-91405 Orsay, France

K. March and L. F. Zagonel

Laboratoire de Physique des Solides, Université Paris Sud XI, UMR 8502 CNRS, F-91405 Orsay, France

\author{
R. Songmuang \\ CEA-CNRS Group “Nanophysique et Semiconducteurs”, 17 Rue des Martyrs, F-38054 Grenoble Cedex 9, France
}

(Received 2 November 2010; revised manuscript received 11 January 2011; published 10 March 2011)

\begin{abstract}
We report the demonstration of an ultra-sensitive Raman probing of single GaN/AIN nanowires (NWs). The high sensitivity of the Raman scattering by longitudinal optical phonon is achieved by using ultraviolet resonant excitation near the energy band-gap of GaN. Structural variations within one single nanowire are evidenced very accurately by strong LO phonons shifts in the UV Raman spectra recorded on different regions of the NW. They are interpreted as a fine probing of the double strain state experienced by GaN, due to the formation of an AlN shell in the bottom part of the NW. The core-shell structure has been confirmed in a statistical way by measuring the average strain in the NWs ensemble thanks to the Raman scattering excited in the visible range. Data have been comprehensively accounted for by considering an axial strain in GaN NW part covered by AlN shell, in the elastic regime, while the top $\mathrm{GaN}$ is relaxed.
\end{abstract}

DOI: 10.1103/PhysRevB.83.115417

PACS number(s): 63.20.kd, 63.22.Gh

\section{INTRODUCTION}

III-nitride semiconductors and their alloys are intensively studied mostly for their optoelectronic applications to light emitting diodes and lasers. III-nitride nanostructures in the form of quantum wells, quantum dots or nanowires have been intensively used in optoelectronic devices operating in the blue to UV spectral range. The 1D geometry of the NWs is well adapted for emission and detection of light since it forms a waveguide for a certain range of NWs diameters. Besides, high quality GaN/AlN NW heterostructures pave the way for the realization of single quantum dot devices and resonant tunneling diode which is an important step toward a variety of III-N based quantum devices relying on axial quantum confinement. ${ }^{1}$ Light sources at the nanometer scale and other sub-wavelength photonic components based on single-crystalline GaN NWs or nanotubes have already been demonstrated. ${ }^{2}$ Ultra-violet photodetectors based on a single GaN NW containing quantum discs have also been reported. ${ }^{3}$ New possibilities for wavelength tuning have been offered with the demonstration of GaN NW-ring lasers. ${ }^{4}$ The unique geometry and properties of semiconductor NWs have motivated their use for new applications, such as nanoscale photodetectors with high polarization degree of detection. ${ }^{5}$

In this paper, we focus on the relation between the coreshell structure and the related strain in functionalized NWs. Induced strain states are indeed known to monitor physical properties of individual as well as large populations of nanoobjects. Several experimental techniques have been combined to deeply investigate structural, morphological, spectroscopic and mechanical behavior of those nano-objects.

One technique of choice for the study of NWs is microRaman ( $\mu$-Raman) spectroscopy which represents an extremely active and exciting field allowing to explore new phenomena at the nanometer scale. Inelastic light scattering spectroscopy has been intensively applied to single GaAs, AlGaAs and InP $\mathrm{NWs}^{6-9}$ with a special attention paid to the polarization, resonance, structural and strain effects. In particular, unusual angular dependencies of the vibrational modes can be evidenced in relation with the highly anisotropic shape of the NWs. Exciton scattering has also been found to be the main effect contributing to the electron-phonon coupling by a resonant Raman scattering study ${ }^{7}$ in single GaAs NWs. The strain study in single NW has recently benefited of original approaches such as the analysis of bent semiconductor $\mathrm{NWs}^{8}$ and uninentional core-shell structures. ${ }^{9}$ Filamentary crystals have much higher crystalline quality with respect to thin films since their interaction with the underlying substrate is reduced and a lower density of defects can thus be achieved. Raman spectroscopy has previously been applied to study the material properties in self-assembled $\mathrm{GaN}$ NWs such as the crystal structure, composition, orientation, mechanical strain, doping, and phonon confinement. ${ }^{10-13}$ One of the main results is the strong influence of the large surfaceto-volume ratio in NWs on the electronic and vibrational properties. Very recently, structural properties of GaN/AlN core-shell nanocolumn heterostructures have been investigated by a combination of different techniques including Raman spectroscopy. ${ }^{14}$ 
To date, however, there are only few reports on Raman studies of individual GaN NWs ${ }^{15-17}$ essentially based on polarization dependent measurements. Besides, to the best of our knowledge, the only available reports are based on non-resonant Raman studies. Pauzauskie et al $^{15}$ investigated single NWs addressing the questions of their crystal structure, growth direction, and radial crystallographic orientation. Livneh et al. ${ }^{16}$ investigated the phonon symmetries and found the Raman intensities to be governed by the symmetry of complex-valued Raman tensors of the individual bands. The highly polarized Raman scattering anisotropy in a single $70 \mathrm{~nm}$ diameter NW has been reported by of E. O. Schäfer-Nolte et al., ${ }^{17}$ with the exclusive observation in the Raman spectra of the $\mathrm{A}_{1}$-symmetry transverse optical phonon. Moreover, further ideas have been recently proposed for the Raman study of single NWs or Nanotubes. ${ }^{18,19}$ It has been shown that strong enhancement of the Raman scattering could occur depending on the NW diameter, excitation wavelength and incident polarization. ${ }^{18}$ The increased sensitivity of this technique by Tip-Enhanced Raman Spectroscopy (TERS) has also been reported for single GaN NW. ${ }^{19}$ Even if near-field Raman scattering (surface-enhanced and tip-enhanced Raman scattering) are still challenging techniques for semiconducting nanostructures studies, recent advances exploiting plasmonic effects are promising in view of the careful analysis of narrowest single NWs on specific substrates. ${ }^{20,21}$

Here, we report on the UV $\mu$-Raman spectroscopy of a single GaN/AlN NW grown by Molecular Beam Epitaxy (MBE). The bottom part of the GaN NW is covered by an AlN shell whereas the top part has a free lateral surface. We show experimentally for the first time that resonant Raman scattering allows to probe the strain variations within a single NW and we estimate the strain exercised by the AlN shell on the GaN core. The results obtained for a single NW have been confirmed by the Raman measurements on a large population of NWs.

\section{EXPERIMENTAL METHODS}

Catalyst-free-GaN NWs were grown by radio frequency Plasma-Assisted Molecular Beam Epitaxy (PA-MBE) on Si (111) substrates under Nitrogen-rich atmosphere at $790^{\circ} \mathrm{C}$. This atmospheric condition results in a self-assembled NW growth at high density, typically $10^{10} \mathrm{~cm}^{-2}$. Further details about the NW growth are given elsewhere. ${ }^{22}$ The NW average length is $1.2 \mu \mathrm{m}$ and the typical diameter is in the 45-90 nm range. The NWs are successively constituted by a GaN base part of approximately $500 \mathrm{~nm}$ (Si doped, $\left.n \sim 2.10^{19} \mathrm{~cm}^{-3}\right)$, followed by a twenty-period AlN $(3 \mathrm{~nm}) /$ $\mathrm{GaN}(5 \mathrm{~nm})$ multiquantum-disc (QDisc) structure and finally covered by a silicon doped GaN top part ( $500 \mathrm{~nm})$.

The NWs were detached from the Si substrates by an ultrasonic bath and then dispersed in ethanol on $\mathrm{Si}$, in such a way that the NW density is reduced to $10^{6} \mathrm{~cm}^{-2}$. A regular array of geometrical marks extending over a millimetric range has been engraved at the surface of the sample by a focused $\mathrm{Ga}^{+}$ion beam (FIB), and has been used as a reference frame to locate any single NW within this area.

The nanowire morphologies, dimensions and orientations were investigated by a number of microscopy techniques including atomic force microscopy (AFM; Veeco - Dimension 3100), scanning electron microscopy (SEM; Zeiss CrossBeam 1540) and scanning transmission electron microscopy (STEM; Jeol). Morphological characterization of single GaN NWs has been performed by AFM working in the tapping mode to minimize probe-to-surface mechanical interactions and thus prevent any displacement of the NW by the tip. Large scale images first enabled to precisely locate individual NWs with respect to FIB marks and small scale ones have then been used to measure the geometrical features of the NWs.

The near-resonance $\mu$-Raman spectroscopy was conducted at room temperature by a Dilor $X Y$ spectrometer coupled to a UV optical microscope, with the exciting laser at $351 \mathrm{~nm}$ $(3.53 \mathrm{eV})$. Among the available laser lines $(335 \mathrm{~nm}, 351 \mathrm{~nm}$ and $364 \mathrm{~nm}$ ), the $351 \mathrm{~nm}$ excitation line is the closest in energy to the GaN bandgap $\left(E_{g}\right)$ and matches the resonance conditions. The power of the laser spot focused on the sample with a $100 \times$ UV objective in a backscattered configuration has been kept low (approximately $1 \mathrm{~mW}$ ) in order to prevent any damage due to heating effects. Our UV Raman setup, equipped with a video camera together with a $X Y$ piezo stage, has a high mechanical stability and a spatial resolution estimated to be approximately $500 \mathrm{~nm}$. A triple blazed grating stage and a UV sensitive CCD cooled at low temperature are included in the setup. The peak fitting procedure assumes Lorentzian-shaped curves and the peak intensities were determined by peak area integration.

\section{EXPERIMENTAL RESULTS}

\section{A. Structural and morphological studies}

The STEM image performed on a single NW and presented in Fig. 1(a) shows that during the deposition of AlN barriers, an AlN shell is formed around the GaN base part of the NW during the growth of the GaN/AIN superlattice located in the middle of the NW. The formation of this shell leads to a diameter change observed in the STEM image. This shell appears systematically in GaN/AlN NW heterostructures because of the high sticking coefficient of Al facilitating the AlN nucleation. ${ }^{23,24}$ The thickness of the shell is about 5-10 nm. For some wires an additional GaN shell of 5-15 nm surrounding the QDisc region and the lower NW part is formed
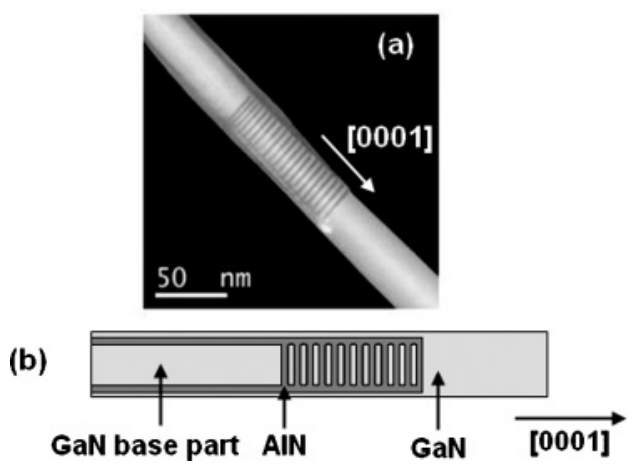

FIG. 1. (a) Dark field STEM image giving evidence for the formation of an AlN shell at the NW surface surrounding the QDisc region and the lower GaN part. (b) Sketch illustrating the internal structure of the NW as evidenced by STEM image. 


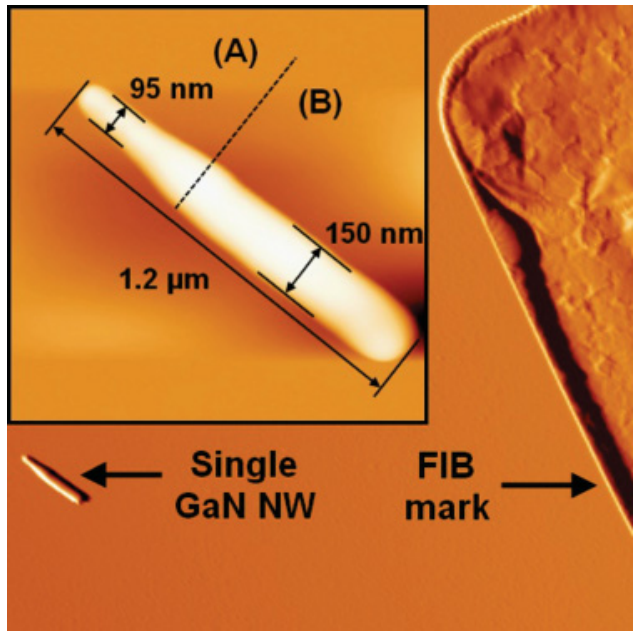

FIG. 2. (Color online) Large scale AFM image $(10 \mu \mathrm{m} \times 10 \mu \mathrm{m})$ showing the individual GaN NW under study on the left and the FIB mark on the right side. Inset: Small scale AFM image $(1.2 \mu \mathrm{m} \times$ $1.2 \mu \mathrm{m})$ of the same NW confirming its non-uniform geometry together with the length and the diameter values at both ends.

during the deposition of the top GaN segment. Fig. 1(b) shows a sketch of the internal structure of the NWs under study.

The large scale AFM amplitude image $(10 \mu \mathrm{m} \times 10 \mu \mathrm{m})$ given in Fig. 2 shows an individual GaN NW located at about $7.5 \mu \mathrm{m}$ from a FIB mark engraved on the Si substrate. A small scale $(1.2 \mu \mathrm{m} \times 1.2 \mu \mathrm{m})$ AFM topographical image of this GaN NW is presented in the inset of Fig. 2. From this image, its length is found to be approximately $1.2 \mu \mathrm{m}$ and its diameter is comprised between $95 \mathrm{~nm}$ at one end and $150 \mathrm{~nm}$ at the other. This NW exhibits a very specific shape and a clear non-uniformity of its diameter with a relative diameter variation of $50 \%$ along the NW axis. This relatively large diameter single NW has been chosen among a large population of studied ones, because of the strong Raman signal collected.

\section{B. Polarization effects}

The Raman spectra of Fig. 3 and Fig. 4 have been acquired from the NW of Fig. 2 with a short acquisition time of $60 \mathrm{~s}$, thanks to the enhanced sensitivity provided by resonant excitation. For this polarization study, laser focus was done approximately in the part of the NW for which the apparent diameter is larger. In Fig. 3, the NW was excited at the same point for each measurement with a constant incident power. Since the NW axis is perpendicular to the incident photon wave-vector and the scattered light being un-analyzed in our experiments, the scattering configuration is denoted by $X(Z,-) \bar{X}$ [respectively $X(Y,-) \bar{X}$ ] when the polarization is along (respectively perpendicular to) the NW axis. Let's recall that $Z$ refers to the direction of $\mathbf{c}$-axis, parallel to the long axis of the NW. These two polarization configurations are labeled $\pi$ (for parallel) and $\sigma$ (for perpendicular) (see Fig. 3). AFM imaging of the microscopic zone surrounding the GaN NW has been performed after the optical characterization and has been used to assess the relative orientation of the polarizer and the NW axes, the regular array of FIB marks on the surface being used as a reference frame. Large scale AFM images

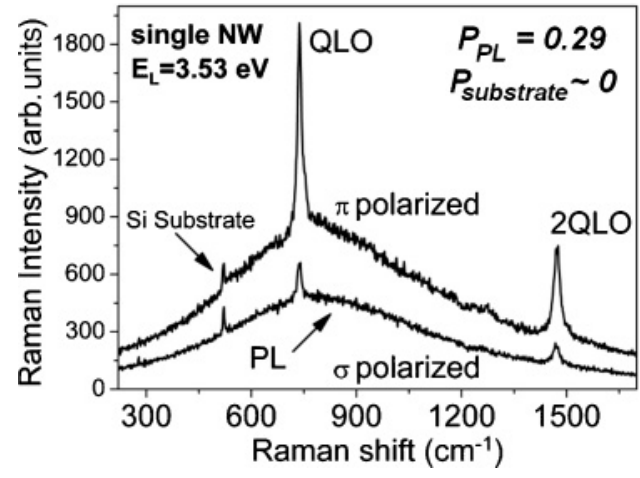

FIG. 3. Room temperature UV Raman spectra of the same GaN NW for the two orientations of the NW axis with respect to the polarization of the incident laser. $\pi$ polarization indicates parallel directions of the polarization of incident light and the NW axis while $\sigma$ polarization refers to the laser polarization direction perpendicular to the NW axis.

also ensure that the $500 \mathrm{~nm}$ diameter laser spot interacts with only one NW at a time and that the optical data are free from coupling effects between individual NWs.

A broad PL band corresponding to the near band-edge (NBE) photo-luminescence (PL) is observed together with strong multiple LO phonon scattering up to second order and a very weak contribution from the underlying Si substrate. The intensity of the overall peaks is clearly larger in the $\pi$ polarized spectrum, except for the Si substrate peaks. For each peak, either phonons or PL, the polarization ratio $P$ is defined as

$$
P=\frac{I_{\pi}-I_{\sigma}}{I_{\pi}+I_{\sigma}}
$$

where $I_{\pi}$ and $I_{\sigma}$ are the integrals of the peak corresponding to the $\pi$ and $\sigma$ polarizations, respectively. We calculated the polarization ratio extracted from the fitting procedure of the two peaks considered here, which are the PL peak and the substrate phonon peak. This leads to the two values of polarization ratio $P_{\mathrm{PL}}=0.29$ and $P_{\text {substrate }} \sim 0$. As expected, the polarization ratio is nearly zero for $\mathrm{Si}$ substrate, for which the intensity of the well known mode at $520 \mathrm{~cm}^{-1}$ is almost constant in the two polarization configurations. The low value of the polarization ratio of the NW is likely due to its large diameter and is in reasonable agreement with the previous study by low temperature micro-PL on a similar sample. ${ }^{25}$ We underline that in our case, due to the strong resonant conditions, this polarization ratio has been measured by polarization control of the incident photons with respect to the NW axis, while emitted photons were analysed by Rigutti et al. in off resonant PL excitation. Note that the experimental value of 0.29 is much lower than the theoretical value of 0.93 evaluated by using the dielectric contrast model. ${ }^{26} \mathrm{It}$ is worthwhile to keep in mind that this model is in good agreement with experimental data obtained by Wang et al. ${ }^{5}$ for large aspect ratio and small diameters $(\sim 10-40 \mathrm{~nm}) \mathrm{NWs}$ contrary to the one investigated here $(\sim 100 \mathrm{~nm})$. In this latter case, the screening of the electric field perpendicular to the NW axis, which is related to the dielectric contrast between the NW and the surrounding air, tends to be weaker than the one expected for NWs with a smaller diameter in 
the $10-40 \mathrm{~nm}$ range. Interestingly, the intensity contrast of the quasi-longitudinal optical (QLO) phonon and its second harmonic (2QLO) measured from the spectra of Fig. 3 is much higher than the PL intensity contrast. Nevertheless, we think that any attribution to polarization effects related to $1 \mathrm{D}$ geometry of the $\mathrm{NW}$ has to be ruled out. Indeed, in the $X(-,-) \bar{X}$ backscattering configuration, $\mathrm{A}_{1}(\mathrm{TO}), \mathrm{E}_{1}(\mathrm{TO})$ and $E_{2}$ are symmetry allowed. Due to resonant scattering, these modes usually dominant in non resonant first-order scattering, no longer appear. On the contrary, scattering by the polar QLO phonon is clearly observed at $738 \mathrm{~cm}^{-1}$, close to the $\mathrm{E}_{1}(\mathrm{LO})$ phonon frequency. In $X(-,-) \bar{X}$ configuration, $\mathrm{QLO}$ mode refers to LO phonon with quasi- $E_{1}$ symmetry and is generally observed in non resonant Raman scattering spectra recorded in the $X(Z, Z) \bar{X}$ polarization configuration. ${ }^{27-29} \mathrm{~A}$ weak contribution of $A_{1}$ symmetry tensor is generally admitted because of an admixture of a small $A_{1}$ component due to the aperture of the focusing lens. We believe that in the $X(Y,-) \bar{X}$ configuration, QLO phonon scattering is not clearly observed in our experiments for symmetry considerations : for phonon wave-vector perpendicular to the c-axis in wurtzite crystals, diagonal $Y Y$ and $Z Z$ components of the electronic susceptibility due to the intraband Fröhlich interaction are different in principle. ${ }^{30} \mathrm{We}$ assume that the $q$-dependent Fröhlich electron-phonon interaction is at the origin of the spectra dominated by strongly peaked, multiple QLO lines in a single GaN NW, due to resonant conditions. In particular this has been correlated for hexagonal $\mathrm{GaN}$ to the large value of the Fröhlich coupling constant, which is around six times larger than that of GaAs. ${ }^{31}$ We believe that the breakdown of wave-vector conservation which leads to the strong resonant scattering in our experiments can be caused by electron wave-vector relaxation processes associated with the ionized $\mathrm{Si}$ impurities. Additionally, the strength of these processes depending critically on resonant conditions, we think that the resonant conditions in the presented experiments are almost ideal and are promising for further studies. Let's remark also that in our NW, the excitonic effects in the intermediate states should be ruled out since they are supposed to be screened by the free electrons. Further works are in progress to clarify this question.

\section{Strain effects in a single nanowire}

We then investigated the homogeneity of structural properties within the NW under study by adopting $\pi$ polarization configuration. Spectra acquired in two different locations of the NW, labeled (A) and (B) in Fig. 2, are presented in Fig. 4. Let's point out that careful positioning of the laser spot within the NW has been achieved by taking advantage of the high stability of $X Y$ piezo stage.

Taking into account the frequency of the QLO phonon $\left(738 \mathrm{~cm}^{-1}\right)$ and the energy of the broad NBE PL $(3.424 \mathrm{eV})$, we attribute the (B) spectrum to the strain free part of the NW. NBE PL of spectrum (A) is broader and situated at higher energy (3.45 eV) than the (B) NBE PL, together with QLO phonon frequency blue-shifted to $747 \mathrm{~cm}^{-1}$. Raman signature of the QDiscs is not present in our experiments due to their small active volume. Raman and PL energy shifts observed in (A) spectrum both indicate that the probed area of the

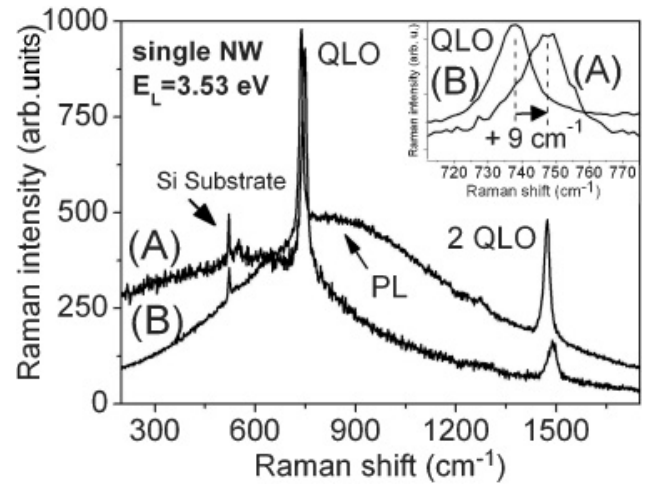

FIG. 4. Raman spectra of the same GaN NW recorded from two different parts. (A) and (B) labels on the figure refers to the Raman spectra acquired from the two different regions identified in Fig. 2. Note that the (A) spectrum signal-to-noise ratio is lower than the (B) spectrum one. Inset shows enlarged view of the QLO phonon spectral region.

GaN NW is strained in comparison with the NW region related to the (B) spectrum. This frequency shift is even more obvious on the second order Raman scattering (2QLO peaks) and found, as expected, twice that of the QLO mode, i.e. $+17 \mathrm{~cm}^{-1}$. Incidentally, the intensity ratio $I_{\mathrm{QLO}} / I_{2 \mathrm{QLO}}$ based on their integrated peak intensities evolves from 1.73 in strain free (B) region to 5.73 in the strained area (A). Resonance effects have to be considered to understand this experimental observation. Resonance at the scattered photon (out-going resonance) in (B) spectrum is located between the first and the second order scattering and consequently does not favour any scattering order. For (A) spectrum, due to compressive strain, the higher energy of electronic transitions leads to an out-going resonance which is shifted below first order scattering energy while resonance at the incident photon (in-going resonance) is improved. Both effects favour strongly the first order scattering in (A) spectrum. This very high sensitivity gives evidence for the acuity of the resonance since third order scattering has never been observed in our experiments. An additional spectrum (not shown) recorded in the middle part of the NW exhibits a double contribution to QLO phonon shape, proving that parts of both regions (A and $\mathrm{B}$ ) of the $\mathrm{NW}$ are simultaneously excited.

\section{Strain effects in an ensemble of nanowires}

We also used the same experimental procedure to perform an average study on a NW ensemble. In Fig. 5, a Raman spectrum acquired with visible excitation (488 nm) of selfassembled vertical NWs grown on $\mathrm{Si}$ is shown.

The strong contribution at $521 \mathrm{~cm}^{-1}$ in the spectra comes from the dominant signal of the substrate. We have been able to get the signature of non-polar $E_{2}$ mode at $567 \mathrm{~cm}^{-1}$ from the NWs which corresponds to the well-known value for stress-free GaN. A slight shoulder appears in the high frequency side of the spectrum. By means of a careful fitting procedure, taking into account unusual background due to the strong silicon signal, we show that this second contribution with $E_{2}$ symmetry is blue-shifted by $7.5 \mathrm{~cm}^{-1}$. It has been attributed to the strained part of the NWs. The broadening 


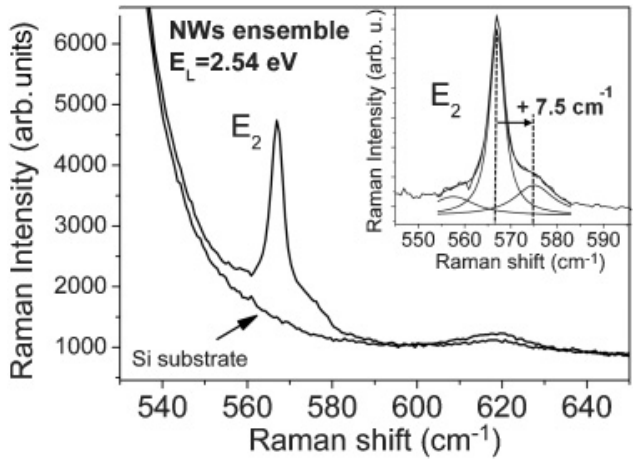

FIG. 5. Visible Raman spectrum of the ensemble of selfassembled GaN NWs together with the reference Raman spectrum of the silicon substrate. Inset presents enlarged view of the $E_{2}$ phonon spectral region together with theoretical curves used for the fitting procedure.

of this contribution is much larger than the $E_{2}$ mode coming from the relaxed $\mathrm{GaN}$ and is attributed to the heterogeneity of strain due to the strong dispersion of the AlN shell thickness in the different NWs observed in STEM characterizations. The intensity ratio between these two $E_{2}$ symmetry Raman contributions leads to $I_{E_{2}}$ (strained) $/ I_{E_{2}}$ (relaxed) $\sim 0.43$, which seems a reasonable value taking into account that the associated volumes are comparable but the axial excitation of the vertical NWs favours the signal from the upper part (relaxed) of the NWs. The lower contribution appearing in the fitting procedure at $560 \mathrm{~cm}^{-1}$ is attributed to the small activation of the forbidden $E_{1}(\mathrm{TO})$ mode due to the objective aperture.

\section{E. Discussion}

To extract some quantitative data in terms of strain from these Raman frequency shifts, we calculated the elastic strain in cylindrical GaN NW strained by AlN shell. It has been pointed out by Hestroffer et al. ${ }^{14}$ that the absolute value of the in-plane strain $\epsilon_{\text {plane }}=\left(\epsilon_{x x}+\epsilon_{y y}\right) / 2$ in the GaN core is one order of magnitude smaller than the absolute value of the axial strain component along the NW axis $\epsilon_{c}$. Therefore, we have used a simplified elastic model neglecting the in-plane strain. In this case, the expression for the actual strain derived from the minimization of the total elastic energy is given by: ${ }^{14}$

$$
\epsilon_{c}=\frac{V^{\text {shell }} c_{33}^{\mathrm{AlN}}}{V^{\text {core }} c_{33}^{\mathrm{GaN}}}\left(\frac{c_{\mathrm{relaxed}}^{\mathrm{GaN}}}{c_{\text {relaxed }}^{\text {AlN }}}\right)^{2}
$$

where $c_{33}$ is the elastic constant of the corresponding material, $V^{\text {shell }}$ and $V^{\text {core }}$ are the shell and core volumes and $c_{\text {relaxed }}$ is the relaxed $c$ lattice parameter of the corresponding material. The GaN elastic coefficients determined by Deger et al. ${ }^{32}$ have been used in the calculation.

Figure 6 presents the calculated strain coefficient as a function of the volumes ratio, and the inset illustrates the corresponding frequency shifts $\delta \omega$ for both $E_{2}$ and QLO modes as a function of this volume ratio, calculated by :

$$
\delta \omega_{E_{2}, \mathrm{QLO}}=b_{E_{2}, \mathrm{QLO}} \cdot \epsilon_{c}
$$

where $b_{i}$ represents the deformation potential of each phonon.

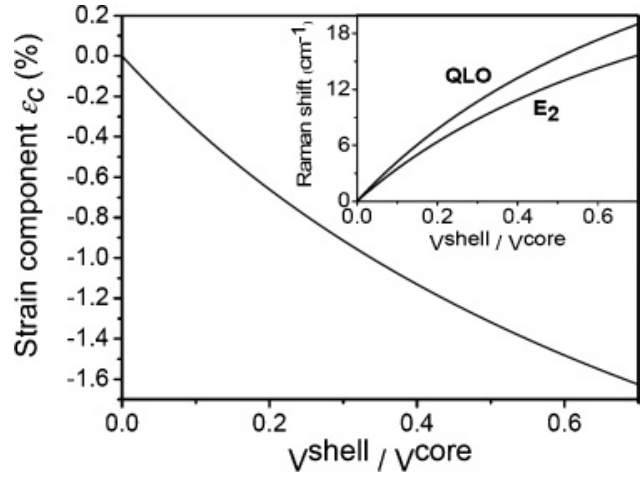

FIG. 6. Axial $\epsilon_{c}$ strain component of the GaN lattice parameter as a function of core-shell volume ratio. Inset gives the Raman frequency shifts of the $E_{2}$ and QLO phonons associated to the corresponding strain.

We used experimental values of these parameters given in Refs. 33 and 34. In this last equation, the in-plane strain has been neglected leading to a direct relation between the Raman shift and the axial strain component. For a large population of NWs, the reported shift of $E_{2}$ phonon corresponds to a value of the strain component of $\epsilon_{c}=-0.78 \%$. In the case of the individual NW under study, the calculated strain component is $\epsilon_{c}=-0.77 \%$. The overall agreement between these two estimations is very good. In both cases, the extracted volume ratios are very similar $(0.24$ and 0.245$)$.

If we assume an hydrostatic strain state in the NW, the tensor components are linked by $\epsilon_{z z}=1.1 \epsilon_{x x}$ leading to the in-plane strain coefficient value of $-0.054 \%$ extracted from the $E_{2}$ mode Raman shift measured on a large number of NWs.

The effective strain state should lie between these two extreme situations. These calculated values are tentative ones and would need to be confirmed by other dedicated experimental techniques such as High Resolution TEM (HRTEM). Nevertheless, to the best of our knowledge, our experimental approach together with these simple models to calculate strain coefficients, give the first insight into the magnitude of strain in single GaN NWs by Raman scattering.

Previous works performed on individual GaN nanopillars of comparable dimensions ${ }^{35}$ have pointed out that the $1 \mathrm{D}$ geometry allows for efficient strain relaxation via the free surface. However, in GaN/AIN core-shell NWs, the strain relaxation takes place only in the radial direction, while the formation of the surrounding AIN shell prevents the strain relaxation along the axial direction of the NW. We find that the strain state induced in a core-shell structure significantly alters the vibrational properties and the band structure of the NW. The present study of the strain distribution within the NWs is of high importance for applications relying on the deformation of NWs such as, for example, probing their piezoelectric response or monitoring the light emission process by tuning the energy bandgap.

\section{CONCLUSION}

In conclusion, we have shown that Raman spectroscopy can be used to probe very sensitively the internal structure of GaN within a single NW. By measuring the UV-resonant 
micro-Raman scattering spectra with high spatial resolution, we evidenced a different strain state in two different segments of a single NW. The structural study indicates that the nonuniformity of the strain is related to the formation of an AlN shell from the bottom of the NWs to the middle of the NWs where a GaN/AIN heterostructure (QDisc/barriers) has been inserted. The strong shift of the LO phonon energy in the NW GaN segment covered by an AlN shell is related to the different strain state with respect to the shell-free segment. We corroborated this single NW analysis with a statistical investigation over a large number of NWs by Raman scattering excited in the visible range, where the strained state within the NWs is revealed by the shifted frequency of the non-polar $E_{2}$ phonon. We finally proposed a phenomenological approach to account for these data by considering the elastic axial strain in $\mathrm{GaN}$ induced by the AlN shell.
*Corresponding author: francois.demangeot@cemes.fr

${ }^{1}$ R. Songmuang and G. Katsaros, E. Monroy, P. Spathis, C. Bougerol, M. Mongillo, and S. De Franceschi, Nano. Lett. 10, 3545 (2010).

${ }^{2}$ D. J. Sirbuly, M. Law, H. Yan and P. Yang, J. Phys. Chem. B , 109, 15190 (2005).

${ }^{3}$ L. Rigutti, M. Tchernycheva, A. De Luna Bugallo, G. Jacopin, F. H. Julien, and L. F. Zagonel, K. March, O. Stephan, M. Kociak, R. Songmuang, Nano Lett. 10, 2939 (2010).

${ }^{4}$ P. J. Pauzauskie, D. J. Sirbuly, P. Yang, Phys. Rev. Lett. 96, 143903 (2006).

${ }^{5}$ J. Wang, M. S. Gudiksen, X. Duan, Y. Cui, and C. M. Lieber, Science 293, 1455 (2001).

${ }^{6}$ I. Zardo, S. Conesa-Boj, F. Peiro, J. R. Morante, J. Arbiol, E. Uccelli, G. Abstreiter, and A. Fontcuberta i Morral, Phys. Rev. B 80, 245324 (2009).

${ }^{7}$ M. Brewster, O. Schimek, S. Reich, and S. Gradečak, Phys. Rev. B 80, 201314(R) (2009).

${ }^{8}$ J. Chen, G. Conache, M.-E. Pistol, S. M. Gray, M. T. Borgström, H. Xu, H. Q. Xu, L. Samuelson, and U. Håkanson, Nano lett. 10, 1280 (2010).

${ }^{9}$ B. Buick, E. Speiser, P. Prete, P. Paiano, N. Lovergine, and W. Richter, Phys. Status Solidi B 247-248, 2027 (2010).

${ }^{10}$ H. Ji, M. Kuball, R. A. Burke, J. M. Redwing, Nanotechnology 18, 445704 (2007).

${ }^{11}$ S. Dhara, S. Chandra, G. Mangamma, S. Kalavathi, P. Sankar, K. G. M. Nair, A. K. Tyagi, C. W. Hsu, C. C. Kuo, L. C. Chen, K. H Chen, K. K. Sriram, Appl. Phys. Lett. 90, 213104 (2007).

${ }^{12}$ K. Jeganathan, R. K. Debnath, R. Meijers, T. Stoica, R. Calarco, D. Grützmacher, and H. Lüth, J. Appl. Phys. 105, 123707 (2009).

${ }^{13}$ C. Bougerol, R. Songmuang, D. Camacho, Y. M. Niquet, R. Mata, A. Cros, and B. Daudin, Nanotechnology 20, 295706 (2009).

${ }^{14}$ K. Hestroffer, R. Mata, D. Camacho, C. Leclere, G. Tourbot, Y. M. Niquet, A. Cros, C. Bougerol, H. Renevier,and B. Daudin, Nanotechnology 21, 415702 (2010).

${ }^{15}$ P. J. Pauzauskie, D. Talaga, K. Seo, P. Yang, and F. LagugnéLabarthet, J. Am. Chem. Soc. 127, 17146 (2005).

${ }^{16}$ T. Livneh, J. Zhang, G. Cheng, and M. Moskovits, Phys. Rev. B 74, 035320 (2006).

${ }^{17}$ E. O. Schäfer-Nolte, T. Stoica, T. Gotschke, F. Limbach, E. Sutter, P. Sutter, and R. Calarco, Appl. Phys. Lett. 96, 091907 (2010).

${ }^{18}$ L. Cao, B. Nabet, and J. E. Spanier, Phys. Rev. Lett. 96, 157402 (2006).
${ }^{19}$ N. Marquestaut, D. Talaga, L. Servant, P. Yang, P. Pauzauskie, and F. Lagugné-Labarthet, J. Raman Spectrosc. 40, 1441 (2009).

${ }^{20}$ L. G. Cançado, A. Jorio, A. Ismach, E. Joselevich, A. Hartschuh, and L. Novotny, Phys. Rev. Lett. 103, 186101 (2009).

${ }^{21}$ X. Deng, G. B. Braun, S. Liu, P. F. Sciortino, B. Koefer, T. Tombler, and M. Moskovits, Nano Lett. 10, 1780 (2010).

${ }^{22}$ R. Songmuang, O. Landré, and B. Daudin, Appl. Phys. Lett. 91, 251902 (2007).

${ }^{23}$ M. Tchernycheva, C. Sartel, G. Cirlin, L. Travers, G. Patriarche, J.-C. Harmand, Le Si Dang, J. Renard, B. Gayral, L. Nevou, F. Julien, Nanotechnology 18, 385306 (2007).

${ }^{24}$ R. Songmuang, T. Ben, B. Daudin, D. González, and E. Monroy, Nanotechnology 21, 295605 (2010).

${ }^{25}$ L. Rigutti, M. Tchernycheva, A. De Luna Bugallo, G. Jacopin, F. H. Julien, F. Furtmayr, M. Stutzmann, M. Eickhoff, R. Songmuang, and F. Fortuna, Phys. Rev. B 81, 045411 (2010).

${ }^{26}$ L. D. Landau, E. M. Lifshitz, L. E. Pitaevskii, Electrodynamics of continuous Media (Pergamon, Oxford 1984).

${ }^{27}$ T. Azuhata, T. Sota, K. Suzuki, and S. Nakamura, J. Phys. Condens. Matter 7, L129 (1995).

${ }^{28}$ F. Demangeot, J. Groenen, J. Frandon, M. A. Renucci, O. Briot, S. Clur, R. L. Aulombard, Appl. Phys. Lett. 72, 2674 (1998).

${ }^{29}$ F. Demangeot, J. Frandon, M. A. Renucci, N. Grandjean, B. Beaumont, J. Massies, P. Gibart, Solid State Commun. 106, 491 (1998).

${ }^{30}$ W. Richter, G. Höhler, Resonant Raman Scattering in Semiconductors, Springer Tracts in Modern Physics, 78 (Springer, Berlin, 1976).

${ }^{31}$ M. Cardona, G. Güntherodt, Light Scattering in Solids II, Topics in Applied Physics, 50 (Springer, Berlin, 1982).

${ }^{32}$ C. Deger, E. Born, H. Angerer, O. Ambacher, M. Stuztmann, J. Hornsteiner, E. Riha, and G. Fischerauer, Appl. Phys. Lett. 72, 2400 (1998).

${ }^{33}$ F. Demangeot, J. Frandon, P. Baulès, F. Natali, F. Semond, J. Massies, Phys. Rev. B 69, 155215 (2004).

${ }^{34}$ V. Darakchieva, T. Paskova, M. Schubert, H. Arwin, P. P. Paskov, B. Monemar, D. Hommel, M. Heuken, J. Off, F. Scholz, B. A. Haskell, P. T. Fini, J. S. Speck, and S. Nakamura, Phys. Rev. B 75, 195217 (2007)

${ }^{35}$ F. Demangeot, J. Gleize, J. Frandon, M. A. Renucci, M. Kuball, D. Peyrade, L. Manin-Ferlazzo, Y. Chen, and N. Grandjean, J. Appl. Phys. 91, 6520 (2002). 